\title{
On Averaging the Best Samples in Evolutionary Computation
}

\author{
Laurent Meunier ${ }^{1,2(\bowtie)}$, Yann Chevaleyre ${ }^{2}$, Jeremy Rapin ${ }^{1}$, \\ Clément W. Royer ${ }^{2}$, and Olivier Teytaud ${ }^{1}$ \\ 1 Facebook Artificial Intelligence Research (FAIR), Paris, France \\ laurentmeunier@fb.com \\ 2 LAMSADE, CNRS, Université Paris-Dauphine, Université PSL, Paris, France
}

\begin{abstract}
Choosing the right selection rate is a long standing issue in evolutionary computation. In the continuous unconstrained case, we prove mathematically that a single parent $\mu=1$ leads to a suboptimal simple regret in the case of the sphere function. We provide a theoretically-based selection rate $\mu / \lambda$ that leads to better progress rates. With our choice of selection rate, we get a provable regret of order $O\left(\lambda^{-1}\right)$ which has to be compared with $O\left(\lambda^{-2 / d}\right)$ in the case where $\mu=1$. We complete our study with experiments to confirm our theoretical claims.
\end{abstract}

\section{Introduction}

In evolutionary computation, the selected population size often depends linearly on the total population size, with a ratio between $1 / 4$ and $1 / 2: 0.270$ is proposed in $[4,5,10]$ suggest $1 / 4$ and $1 / 2$. However, some sources [8] recommend a lower value $1 / 7$. Experimental results in [16] and theory in [9] together suggest a ratio $\min (d, \lambda / 4)$ with $d$ the dimension, i.e. keep a population size at most the dimension. [12] suggests to keep increasing $\mu$ besides that limit, but slowly enough so that rule $\mu=\min (d, \lambda / 4)$ would be still nearly optimal. Weighted recombination is common [1], but not with a clear gap when compared to truncation ratios [11], except in the case of large population size [17]. There is, overall, limited theory around the optimal choice of $\mu$ for optimization in the continuous setting. In the present paper, we focus on a simple case (sphere function and single epoch), but prove exact theorems. We point out that the single epoch case is important by itself - this is fully parallel optimization $[2,6,13,14]$. Experimental results with a publicly available platform support the approach.

\section{Theory}

We consider the case of a single batch of evaluated points. We generate $\lambda$ points according to some probability distribution. We then select the $\mu$ best and average them. The result is our approximation of the optimum. This is therefore an extreme case of evolutionary algorithm, with a single population; this is commonly used for e.g. hyperparameter search in machine learning $[3,6]$, though in most cases with the simplest case $\mu=1$.

(C) Springer Nature Switzerland AG 2020

T. Bäck et al. (Eds.): PPSN 2020, LNCS 12270, pp. 661-674, 2020.

https://doi.org/10.1007/978-3-030-58115-2_46 


\subsection{Outline}

We consider the optimization of the simple function $x \mapsto\|x-y\|^{2}$ for an unknown $y \in \mathcal{B}(0, r)$. In Sect. 2.2 we introduce notations. In Sect. 2.3 we analyze the case of random search uniformly in a ball of radius $h$ centered on $y$. We can, therefore, exploit the knowledge of the optimum's position and assume that $y=0$. We then extend the results to random search in a ball of radius $r$ centered on 0 , provided that $r>\|y\|$ and show that results are essentially the same up to an exponentially decreasing term (Sect. 2.4).

\subsection{Notations}

We are interested in minimizing the function $f: x \in \mathbb{R}^{d} \mapsto\|x-y\|^{2}$ for a fixed unknown $y$ in parallel one-shot black box optimization, i.e. we sample $\lambda$ points $X_{1}, \ldots, X_{\lambda}$ from some distribution $\mathcal{D}$ and we search for $x^{\star}=\arg \min _{x} f(x)$. In what follows we will study the sampling from $\mathcal{B}(0, r)$, the uniform distribution on the $\ell_{2}$-ball of radius $r$; w.l.o.g. $\mathcal{B}(y, r)$ will also denote the $\ell_{2}$-ball centered in $y$ and of radius $r$.

We are interested in comparing the strategy " $\mu$-best" vs "1-best". We denote $X_{(1)}, \ldots, X_{(\lambda)}$, the sorted values of $X_{i}$ i.e. $(1), \ldots,(\lambda)$ are such that $f\left(X_{(1)}\right) \leq \ldots \leq$ $f\left(X_{(\lambda)}\right)$. The " $\mu$-best" strategy is to return $\bar{X}_{(\mu)}=\frac{1}{\mu} \sum_{i=1}^{\mu} X_{(i)}$ as an estimate of the optimum and the "1-best" is to return $X_{(1)}$. We will hence compare : $\mathbb{E}\left[f\left(\bar{X}_{(\mu)}\right)\right]$ and $\mathbb{E}\left[f\left(X_{(1)}\right)\right]$. We recall the definition of the gamma function $\Gamma: \forall z>0, \Gamma(z)=\int_{0}^{\infty} t^{z-1} e^{-t} d t$, as well as the property $\Gamma(z+1)=z \Gamma(z)$.

\subsection{When the Center of the Distribution is also the Optimum}

In this section we assume that $y=0$ (i.e. $f(x)=\|x\|^{2}$ ) and consider sampling in $\mathcal{B}(0, r) \subset \mathbb{R}^{d}$. In this simple case, we show that keeping the best $\mu>1$ sampled points is asymptotically a better strategy than selecting a single best point. The choice of $\mu$ will be discussed in Sect. 2.4.

Theorem 1. For all $\lambda>\mu \geq 2$ and $d \geq 2, r>0$, for $f(x)=\|x\|^{2}$,

$$
\mathbb{E}_{X_{1}, \ldots, X_{\lambda} \sim \mathcal{B}(0, r)}\left[f\left(\bar{X}_{(\mu)}\right)\right]<\mathbb{E}_{X_{1}, \ldots, X_{\lambda} \sim \mathcal{B}(0, r)}\left[f\left(X_{(1)}\right)\right] .
$$

To prove this result, we will compute the value of $\mathbb{E}\left[f\left(\bar{X}_{(\mu)}\right)\right]$ for all $\lambda$ and $\mu$. The following lemma gives a simple way of computing the expectation of a function depending only on the norm of its argument.

Lemma 2. Let $d \in \mathbb{N}^{*}$. Let $X$ be drawn uniformly in $\mathcal{B}(0, r)$ the d-dimensional ball of radius $r$. Then for any measurable function $g: \mathbb{R} \rightarrow \mathbb{R}$, we have

$$
\mathbb{E}_{X \sim \mathcal{B}(0, r)}[g(\|X\|)]=\frac{d}{r^{d}} \int_{0}^{r} g(\alpha) \alpha^{d-1} d \alpha
$$

In particular, we have $\mathbb{E}_{X \sim \mathcal{B}(0, r)}\left[\|X\|^{2}\right]=\frac{d}{d+2} \times r^{2}$. 
Proof. Let $V(r, d)$ be the volume of a ball of radius $r$ in $\mathbb{R}^{d}$ and $S(r, d)$ be the surface of a sphere of radius $r$ in $\mathbb{R}^{d}$. Then $\forall r>0, V(r, d)=\frac{\pi^{d / 2}}{\Gamma\left(\frac{d}{2}+1\right)} r^{d}$ and $S(r, d-1)=\frac{2 \pi^{d / 2}}{\Gamma\left(\frac{d}{2}\right)} r^{d-1}$. Let $g: \mathbb{R} \rightarrow \mathbb{R}$ be a continuous function. Then:

$$
\begin{aligned}
\mathbb{E}_{X \sim \mathcal{B}(0, r)}[g(\|X\|)] & =\frac{1}{V(r, d)} \int_{x:\|x\| \leq r} g(\|x\|) d x \\
& =\frac{1}{V(r, d)} \int_{\alpha=0}^{r} \int_{\theta:\|\theta\|=\alpha} g(\alpha) d \theta d \alpha \\
& =\frac{1}{V(r, d)} \int_{\alpha=0}^{r} g(\alpha) S(\alpha, d-1) d \alpha \\
& =\frac{S(1, d-1)}{V(r, d)} \int_{\alpha=0}^{r} g(\alpha) \alpha^{d-1} d \alpha=\frac{d}{r^{d}} \int_{\alpha=0}^{r} g(\alpha) \alpha^{d-1} d \alpha
\end{aligned}
$$

So, $\mathbb{E}_{X \sim \mathcal{B}(r)}\left[\|X\|^{2}\right]=\frac{d}{r^{d}} \int_{\alpha=0}^{r} \alpha^{2} \alpha^{d-1} d \alpha$

$$
=\frac{d}{r^{d}}\left[\frac{\alpha^{d+2}}{d+2}\right]_{0}^{r}=\frac{d}{d+2} r^{2} .
$$

We now use the previous lemma to compute the expected regret [7] of the average of the $\mu$ best points conditionally to the value of $f\left(X_{(\mu+1)}\right)$. The trick of the proof is that, conditionally to $f\left(X_{(\mu+1)}\right)$, the order of $X_{(1)}, \ldots, X_{(\mu)}$ has no influence over the average. Computing the expected regret conditionally to $f\left(X_{(\mu+1)}\right)$ thus becomes straightforward.

Lemma 3. For all $d>0, r^{2}>h>0$ and $\lambda>\mu \geq 1$, for $f(x)=\|x\|^{2}$,

$$
\mathbb{E}_{X_{1}, \ldots, X_{\lambda} \sim \mathcal{B}(y, r)}\left[f\left(\bar{X}_{(\mu)}\right) \mid f\left(X_{(\mu+1)}\right)=h\right]=\frac{h}{\mu} \times \frac{d}{d+2} .
$$

Proof. Let us first compute $\mathbb{E}\left[f\left(\bar{X}_{(\mu)}\right) \mid f\left(X_{(\mu+1)}\right)=h\right]$. Note that for any function $g: \mathbb{R}^{d} \rightarrow \mathbb{R}$ and distribution $\mathcal{D}$, we have

$$
\begin{aligned}
\mathbb{E}_{X_{1} \ldots X_{\lambda} \sim \mathcal{D}} & {\left[g\left(\bar{X}_{(\mu)}\right) \mid f\left(X_{(\mu+1)}\right)=h\right] } \\
& =\mathbb{E}_{X_{1} \ldots X_{\mu} \sim \mathcal{D}}\left[g\left(\frac{1}{\mu} \sum_{i=1}^{\mu} X_{i}\right) \mid X_{1} \ldots X_{\mu} \in\{x: f(x) \leq h\}\right] \\
& =\mathbb{E}_{X_{1} \ldots X_{\mu} \sim \mathcal{D}_{h}}\left[g\left(\frac{1}{\mu} \sum_{i=1}^{\mu} X_{i}\right)\right]
\end{aligned}
$$


where $\mathcal{D}_{h}$ is the restriction of $\mathcal{D}$ to the level set $\{x: f(x) \leq h\}$. In our setting, we have $\mathcal{D}=\mathcal{B}(0, r)$ and $\mathcal{D}_{h}=\mathcal{B}(0, \sqrt{h})$. Therefore,

$$
\begin{aligned}
\mathbb{E}_{X_{1}, \ldots, X_{\lambda} \sim \mathcal{B}(0, r)} & {\left[f\left(\bar{X}_{(\mu)}\right) \mid f\left(X_{(\mu+1)}\right)=h\right] } \\
& =\mathbb{E}_{X_{1}, \ldots, X_{\lambda} \sim \mathcal{B}(0, r)}\left[\left\|\bar{X}_{(\mu)}\right\|^{2} \mid f\left(X_{(\mu+1)}\right)=h\right] \\
& =\mathbb{E}_{X_{1} \ldots X_{\mu} \sim \mathcal{B}(0, \sqrt{h})}\left[\left\|\frac{1}{\mu} \sum_{i=1}^{\mu} X_{i}\right\|^{2}\right] \\
& =\frac{1}{\mu^{2}} \mathbb{E}_{X_{1} \ldots X_{\mu} \sim \mathcal{B}(0, \sqrt{h})}\left[\sum_{i, j=1}^{\mu} X_{i}^{T} X_{j}\right] \\
& =\frac{1}{\mu^{2}} \sum_{i, j=1, i \neq j}^{\mu} \mathbb{E}_{X_{i} \ldots X_{j} \sim \mathcal{B}(0, \sqrt{h})}\left[X_{i}^{T} X_{j}\right] \\
& +\frac{1}{\mu^{2}} \sum_{i=1}^{\mu} \mathbb{E}_{X_{i} \sim \mathcal{B}(0, \sqrt{h})}\left[\left\|X_{i}\right\|^{2}\right]=\frac{1}{\mu} \mathbb{E}_{X \sim \mathcal{B}(0, \sqrt{h})}\left[\|X\|^{2}\right] .
\end{aligned}
$$

By Lemma 2, we have: $\mathbb{E}_{X \sim \mathcal{B}(0, \sqrt{h})}\left[\|X\|^{2}\right]=\frac{d}{d+2} h$. Hence $\mathbb{E}_{X_{1}, \ldots, X_{\lambda} \sim \mathcal{B}(0, r)}\left[f\left(\bar{X}_{(\mu)}\right) \mid f\left(X_{(\mu+1)}\right)=h\right]=\frac{d}{d+2} \frac{h}{\mu}$.

The result of Lemma 3 shows that $\mathbb{E}\left[f\left(\bar{X}_{(\mu)}\right) \mid f\left(X_{(\mu+1)}\right)=h\right]$ depends linearly on $h$. We now establish a similar dependency for $\mathbb{E}\left[f\left(X_{(1)}\right) \mid f\left(X_{(\mu+1)}\right)=h\right]$.

Lemma 4. For $d>0, h>0, \lambda>\mu \geq 1$, and $f(x)=\|x\|^{2}$,

$$
\mathbb{E}_{X_{1}, \ldots, X_{\lambda} \sim \mathcal{B}(0, r)}\left[f\left(X_{(1)}\right) \mid f\left(X_{(\mu+1)}\right)=h\right]=h \frac{\Gamma\left(\frac{d+2}{d}\right) \Gamma(\mu+1)}{\Gamma(\mu+1+2 / d)} .
$$

Proof. First note that using the same argument as in Lemma $3, \forall \beta \in(0, h]$ :

$$
\begin{aligned}
\mathbb{P}_{X_{1} \ldots X_{\lambda} \sim \mathcal{B}(0, \sqrt{h})} & {\left[f\left(X_{(1)}\right)>\beta \mid f\left(X_{(\mu+1)}\right)=h\right] } \\
& =\mathbb{P}_{X_{1} \ldots X_{\mu} \sim \mathcal{B}(0, \sqrt{h})}\left[f\left(X_{1}\right)>\beta, \ldots, f\left(X_{\mu}\right)>\beta\right] \\
& =\mathbb{P}_{X \sim \mathcal{B}(0, \sqrt{h})}[f(X)>\beta]^{\mu} .
\end{aligned}
$$

Recall that the volume of a $d$-dimensional ball of radius $r$ is proportional to $r^{d}$. Thus, we get:

$$
\mathbb{P}_{X \sim \mathcal{B}(0, \sqrt{h})}[f(X)<\beta]=\frac{\sqrt{\beta}^{d}}{\sqrt{h}^{d}}=\left(\frac{\beta}{h}\right)^{\frac{d}{2}} .
$$


It is known that for every positive random variable $X, \mathbb{E}(X)=\int_{0}^{\infty} \mathbb{P}(X>\beta) d \beta$. Therefore:

$$
\begin{aligned}
\mathbb{E}_{S}\left[f\left(X_{(1)}\right) \mid f\left(X_{(\mu+1)}\right)=h\right] & =\int_{0}^{h} \mathbb{P}\left[f\left(X_{(1)}\right)>\beta \mid f\left(X_{(\mu+1)}\right)=h\right] d \beta \\
& =\int_{0}^{h}\left(1-\left(\frac{\beta}{h}\right)^{\frac{d}{2}}\right)^{\mu} d \beta \\
& =h \int_{0}^{1}\left(1-u^{\frac{d}{2}}\right)^{\mu} d u \\
& =h \frac{2}{d} \int_{0}^{1}(1-t)^{\mu} t^{2 / d-1} d t=h \frac{\Gamma\left(\frac{d+2}{d}\right) \Gamma(\mu+1)}{\Gamma(\mu+1+2 / d)}
\end{aligned}
$$

To obtain the last equality, we identify the integral with the beta function of parameters $\mu+1$ and $\frac{2}{d}$.

We now directly compute $\mathbb{E}_{X_{1}, \ldots, X_{\lambda} \sim \mathcal{B}(0, r)}\left[f\left(X_{(1)}\right)\right]$.

Lemma 5. For all $d>0, \lambda>0$ and $r>0$ :

$$
\mathbb{E}_{X_{1}, \ldots, X_{\lambda} \sim \mathcal{B}(0, r)}\left[f\left(X_{(1)}\right)\right]=r^{2} \frac{\Gamma\left(\frac{d+2}{d}\right) \Gamma(\lambda+1)}{\Gamma(\lambda+1+2 / d)} .
$$

Proof. As in Lemma 4, we have for any $\beta \in\left(0, r^{2}\right]$ :

$$
\begin{aligned}
\mathbb{P}_{X_{1} \ldots X_{\lambda} \sim \mathcal{B}(0, r)}\left[f\left(X_{(1)}\right)>\beta\right] & =\mathbb{P}_{X_{1} \ldots X_{\lambda} \sim \mathcal{B}(0, r)}\left[f\left(X_{1}\right)>\beta, \ldots, f\left(X_{\lambda}\right)>\beta\right] \\
& =\mathbb{P}_{X \sim \mathcal{B}(0, r)}[f(X)>\beta]^{\lambda} \\
& =\left(\frac{\sqrt{\beta}}{r}\right)^{d} .
\end{aligned}
$$

The result then follows by reasoning as in the proof of Lemma 4 .

By combining the results above, we obtain the exact formula for $\mathbb{E}\left[f\left(\bar{X}_{(\mu)}\right)\right]$.

Theorem 6. For all $d>0, r>0$ and $\lambda>\mu \geq 1$ :

$$
\mathbb{E}_{X_{1} \ldots X_{\lambda} \sim \mathcal{B}(0, r)}\left[f\left(\bar{X}_{(\mu)}\right)\right]=\frac{r^{2} d \times \Gamma(\lambda+1) \Gamma(\mu+1+2 / d)}{\mu(d+2) \Gamma(\mu+1) \Gamma(\lambda+1+2 / d)} .
$$


Proof. The proof follows by applying our various lemmas and integrating over all possible values for $h$. We have:

$$
\begin{aligned}
& \mathbb{E}_{X_{1} \ldots X_{\lambda} \sim \mathcal{B}(0, r)}\left[f\left(\bar{X}_{(\mu)}\right)\right] \\
& =\mathbb{E}\left[\mathbb{E}\left[f\left(\bar{X}_{(\mu)}\right) \mid f\left(X_{(\mu+1)}\right)\right]\right] \\
& =\frac{1}{\mu} \frac{d}{d+2} \mathbb{E}\left[f\left(X_{(\mu+1)}\right)\right] \text { by Lemma } 3 \\
& =\frac{1}{\mu} \frac{d}{d+2} \frac{\Gamma(\mu+1+2 / d)}{\Gamma(\mu+1) \Gamma\left(\frac{d+2}{d}\right)} \mathbb{E}\left[\mathbb{E}\left[f\left(X_{(1)}\right) \mid f\left(X_{(\mu+1)}\right)\right]\right] \text { by Lemma } 4 \\
& =\frac{1}{\mu} \frac{d}{d+2} \frac{\Gamma(\mu+1+2 / d)}{\Gamma(\mu+1) \Gamma\left(\frac{d+2}{d}\right)} \mathbb{E}\left[f\left(X_{(1)}\right)\right] \\
& =\frac{r^{2} d \times \Gamma(\lambda+1) \Gamma(\mu+1+2 / d)}{\mu(d+2) \Gamma(\mu+1) \Gamma(\lambda+1+2 / d)} \text { by Lemma } 5 .
\end{aligned}
$$

We have checked experimentally the result of Theorem 9 (see Fig. 1): the result of Theorem 1 follows from Theorem 9 since for $d \geq 2, \lambda$ and $r$ fixed, $\mathbb{E}\left[f\left(\bar{X}_{(\mu)}\right)\right]$ is strictly decreasing in $\mu$. In addition, we can obtain asymptotic progress rates:

Corollary 7. Consider $d>0$. When $\lambda \rightarrow \infty$, we have

$$
\mathbb{E}_{X_{1} \ldots X_{\lambda} \sim \mathcal{B}(0, r)}\left[f\left(\bar{X}_{(\mu)}\right)\right] \sim \lambda^{-\frac{2}{d}} \frac{r^{2} d \times \Gamma(\mu+1+2 / d)}{\mu(d+2) \Gamma(\mu+1)},
$$

while if $\lambda \rightarrow \infty$ and $\mu(\lambda) \rightarrow \infty, \mathbb{E}_{X_{1} \ldots X_{\lambda} \sim \mathcal{B}(0, r)}\left[f\left(\bar{X}_{(\mu(\lambda))}\right)\right] \sim r^{2} \frac{d}{d+2} \frac{\mu(\lambda)^{\frac{2}{d}-1}}{\lambda^{\frac{2}{d}}}$. As a result, $\forall c \in(0,1), \mathbb{E}\left(f\left(\bar{X}_{(\lfloor c \lambda\rfloor)}\right)\right) \in \Theta\left(\frac{1}{\lambda}\right)$ and $\mathbb{E}\left(f\left(X_{(1)}\right)\right) \in \Theta\left(\frac{1}{\lambda^{2 / d}}\right)$.

Proof. We recall the Stirling equivalent formula for the gamma function: when $z \rightarrow \infty$,

$$
\Gamma(z)=\sqrt{\frac{2 \pi}{z}}\left(\frac{z}{e}\right)^{z}\left(1+O\left(\frac{1}{z}\right)\right) .
$$

Using this approximation, we get the expected results.

This result shows that by keeping a single parent, we lose more than a constant factor: the progress rate is significantly impacted. Therefore it is preferable to use more than one parent.

\subsection{Convergence When the Sampling is not Centered on the Optimum}

So far we treated the case where the center of the distribution and the optimum are the same. We now assume that we sample from the distribution $\mathcal{B}(0, r)$ and that the function $f$ is $f(x)=\|x-y\|^{2}$ with $\|y\| \leq r$. We define $\epsilon=\frac{\|y\|}{r}$. 
Lemma 8. Let $r>0, d>0, \lambda>\mu \geq 1$, we have:

$$
\mathbb{P}_{X_{1} \ldots X_{\lambda} \sim \mathcal{B}(0, r)}\left(f\left(X_{(\mu+1)}\right)>(1-\epsilon)^{2} r^{2}\right)=\mathbb{P}_{U \sim B\left(\lambda,(1-\epsilon)^{d}\right)}(U \leq \mu),
$$

where $B(\lambda, p)$ is a binomial law of parameters $\lambda$ and $p$.

Proof. We have $f\left(X_{(\mu+1)}\right)>(1-\epsilon) r \Longleftrightarrow \sum_{i=1}^{\lambda} \mathbb{1}_{\left\{f\left(X_{i}\right) \leq(1-\epsilon)^{2} r^{2}\right\}} \leq \mu$ since $\mathbb{1}_{\left\{f\left(X_{i}\right) \leq(1-\epsilon)^{2} r^{2}\right\}}$ are independent Bernoulli variables of parameter $(1-\epsilon)^{d}$, hence the result.

Using Lemma 8, we now get lower and upper bounds on $\mathbb{E}\left[f\left(X_{(\mu+1)}\right)\right]$ :

Theorem 9. Consider $d>0, r>0, \lambda>\mu \geq 1$. The expected value of $f\left(\bar{X}_{(\mu)}\right)$ satisfies both

$$
\begin{aligned}
\mathbb{E}_{X_{1} \ldots X_{\lambda} \sim \mathcal{B}(0, r)}\left[f\left(\bar{X}_{(\mu)}\right)\right] \leq & 4 r^{2} \mathbb{P}_{U \sim B\left(\lambda,(1-\epsilon)^{d}\right)}(U \leq \mu) \\
+ & \frac{r^{2} d \times \Gamma(\lambda+1) \Gamma(\mu+1+2 / d)}{\mu(d+2) \Gamma(\mu+1) \Gamma(\lambda+1+2 / d)} \\
\text { and } \quad \mathbb{E}_{X_{1} \ldots X_{\lambda} \sim \mathcal{B}(0, r)}\left[f\left(\bar{X}_{(\mu)}\right)\right] & \geq \frac{r^{2} d \times \Gamma(\lambda+1) \Gamma(\mu+1+2 / d)}{\mu(d+2) \Gamma(\mu+1) \Gamma(\lambda+1+2 / d)} .
\end{aligned}
$$

Proof.

$$
\begin{aligned}
\mathbb{E}\left[f\left(\bar{X}_{(\mu)}\right)\right] & =\mathbb{E}\left(f\left(\bar{X}_{(\mu)}\right) \mid f\left(X_{(\mu+1)}\right) \geq(1-\epsilon)^{2} r^{2}\right) \mathbb{P}\left(f\left(X_{(\mu+1)}\right) \geq(1-\epsilon)^{2} r^{2}\right) \\
& +\mathbb{E}\left(f\left(\bar{X}_{(\mu)}\right) \mid f\left(X_{(\mu+1)}\right)<(1-\epsilon)^{2} r^{2}\right) \mathbb{P}\left(f\left(X_{(\mu+1)}\right)<(1-\epsilon)^{2} r^{2}\right) .
\end{aligned}
$$

In this Bayes decomposition, we can bound the various terms as follows:

$$
\begin{aligned}
\mathbb{E}\left(f\left(\bar{X}_{(\mu)}\right) \mid f\left(X_{(\mu+1)}\right)\right. & \left.\geq(1-\epsilon)^{2} r^{2}\right) \leq 4 r^{2} \\
\mathbb{P}\left(f\left(X_{(\mu+1)}\right)\right. & \left.\geq(1-\epsilon)^{2} r^{2}\right) \leq 1 \\
\mathbb{E}\left[f\left(\bar{X}_{(\mu)}\right) \mid f\left(X_{(\mu+1)}\right)\right. & \left.<(1-\epsilon)^{2} r^{2}\right] \leq \frac{r^{2} d \times \Gamma(\lambda+1) \Gamma(\mu+1+2 / d)}{\mu(d+2) \Gamma(\mu+1) \Gamma(\lambda+1+2 / d)} .
\end{aligned}
$$

Combining these equations yields the first (upper) bound. The second (lower) bound is deduced from the centered case (i.e. when the distribution is centered on the optimum) as in the previous section.

Figure 2 gives an illustration of the bounds. Until $\mu \simeq(1-\epsilon)^{d} \lambda$, the centered and non centered case coincide when $\lambda \rightarrow \infty$ : in this case, we can have a more precise asymptotic result for the choice of $\mu$.

Theorem 10. Consider $d>0, r>0$ and $y \in \mathbb{R}^{d}$. Let $\epsilon=\frac{\|y\|}{r} \in[0,1)$ and $f(x)=\|x-y\|^{2}$. When using $\mu=\lfloor c \lambda\rfloor$ with $0<c<(1-\epsilon)^{d}$, we get as $\lambda \rightarrow \infty$, for a fixed $d$,

$$
\mathbb{E}_{X_{1} \ldots X_{\lambda} \sim \mathcal{B}(0, r)}\left[f\left(\bar{X}_{(\mu)}\right)\right]=\frac{d r^{2} c^{2 / d-1}}{(d+2) \lambda}+o\left(\frac{1}{\lambda}\right)
$$


Proof. Let $\mu_{\lambda}=\lfloor c \lambda\rfloor$ with $0<c<(1-\epsilon)^{d}$. We immediately have from Hoeffding's concentration inequality:

$$
\mathbb{P}_{U \sim B\left(\lambda,(1-\epsilon)^{d}\right)}\left(U \leq \mu_{\lambda}\right) \in o\left(\frac{1}{\lambda}\right)
$$

when $\lambda \rightarrow \infty$. From Corollary 7 , we also get:

$$
\frac{r^{2} d \times \Gamma(\lambda+1) \Gamma\left(\mu_{\lambda}+1+2 / d\right)}{\mu_{\lambda}(d+2) \Gamma\left(\mu_{\lambda}+1\right) \Gamma(\lambda+1+2 / d)} \sim \frac{d r^{2} c^{2 / d-1}}{(d+2) \lambda} .
$$

Using the inequalities of Theorem 9, we obtain the desired result.

The result of Theorem 10 shows that a convergence rate $O\left(\lambda^{-1}\right)$ can be attained for the $\mu$-best approach with $\mu>1$. The rate for $\mu=1$ is $\Theta\left(\lambda^{-2 / d}\right)$, proving that the $\mu$-best approach leads asymptotically to a better estimation of the optimum. If we consider the problem $\min _{\mu} \max _{y:\|y\| \leq \epsilon r} \mathbb{E}\left[f_{y}\left(\bar{X}_{(\mu)}\right)\right]$ with $f_{y}$ the objective function $x \mapsto\|x-y\|^{2}$, then $\mu=\lfloor c \lambda\rfloor$ with $0<c<(1-\epsilon)^{d}$ achieves the $O\left(\lambda^{-1}\right)$ progress rate.

All the results we proved in this section are easily extendable to strongly convex quadratic functions. For larger class of functions, it is less immediate, and left as future work.

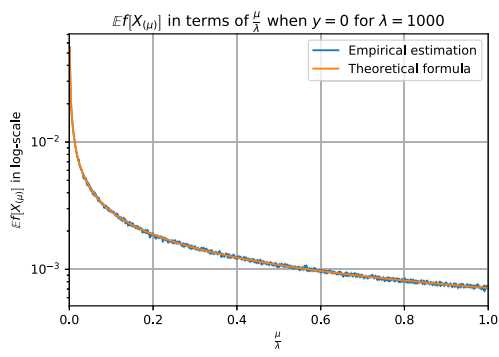

Fig. 1. Centered case: validation of the theoretical formula for $\mathbb{E}_{X_{1} \ldots X_{\lambda} \sim \mathcal{B}(0, r)}\left[f\left(\bar{X}_{(\mu)}\right)\right]$ when $y=0$ from Theorem 6 for $d=5, \lambda=1000$ and $R=1$. 1000 samples have been drawn to estimate the expectation. The two curves overlap, showing agreement between theory and practice.

\subsection{Using Quasi-convexity}

The method above was designed for the sphere function, yet its adaptation to other quadratic convex functions is straightforward. On the other hand, our reasoning might break down when applied to multimodal functions. We thus consider an adaptive strategy to define $\mu$. A desirable property to a $\mu$-best approach is that the level-sets of the functions are convex. A simple workaround is to choose $\mu$ maximal such that there is a quasi-convex function which is identical 
to $f$ on $\left\{X_{(1)}, \ldots, X_{(\mu)}\right\}$. If the objective function is quasi-convex on the convex hull of $\left\{X_{(1)}, \ldots, X_{(\tilde{\mu})}\right\}$ with $\tilde{\mu} \leq \lambda$, then: for any $i \leq \tilde{\mu}, X_{(i)}$ is on the frontier (denoted $\partial$ ) of the convex hull of $\left\{X_{(1)}, \ldots, X_{(i)}\right\}$ and the value

$$
h=\max \left\{i \in[1, \lambda], \forall j \leq i, X_{(j)} \in \partial\left[\operatorname{ConvexHull}\left(X_{(1)}, \ldots, X_{(j)}\right)\right]\right\}
$$

verifies $h \geq \tilde{\mu}$ so that $\mu=\min (h, \tilde{\mu})$ is actually equal to $\tilde{\mu}$. As a result:

- in the case of the sphere function, or any quasi-convex function, if we set $\tilde{\mu}=$ $\left\lfloor\lambda(1-\epsilon)^{d}\right\rfloor$, using $\mu=\min (h, \tilde{\mu})$ leads to the same value of $\mu=\tilde{\mu}=\left\lfloor\lambda(1-\epsilon)^{d}\right\rfloor$. In particular, we preserve the theoretical guarantees of the previous sections for the sphere function $x \mapsto\|x-y\|^{2}$.

- if the objective function is not quasi-convex, we can still compute the quantity $h$ defined above, but we might get a $\mu$ smaller than $\tilde{\mu}$. However, this strategy remains meaningful at it prevents from keeping too many points when the function is "highly" non-quasi-convex.

\section{$3 \quad$ Experiments}
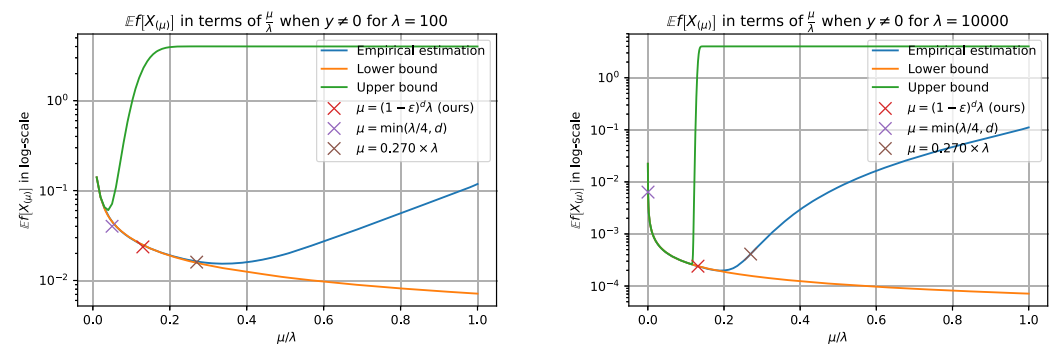

Fig. 2. Non centered case: validation of the theoretical bounds for $\mathbb{E}_{X_{1} \ldots X_{\lambda} \sim \mathcal{B}(0, r)}\left[f\left(\bar{X}_{(\mu)}\right)\right]$ when $\|y\|=\frac{R}{3}$ (i.e. $\epsilon=\frac{1}{3}$ ) from Theorem 9 for $d=5$ and $R=1$. We implemented $\lambda=100$ and $\lambda=10000$. 10000 samples have been drawn to estimate the expectation. We see that such a value for $\mu$ is a good approximation of the minimum of the empirical values: we can thus recommend $\mu=\left\lfloor\lambda(1-\epsilon)^{d}\right\rfloor$ when $\lambda \rightarrow \infty$. We also added some classical choices of values for $\mu$ from literature: when $\lambda \rightarrow \infty$, our method performs the best.

To validate our theoretical findings, we first compare the formulas obtained in Theorems 6 and 9 with their empirical estimates. We then perform larger scale experiments in a one-shot optimization setting.

\subsection{Experimental Validation of Theoretical Formulas}

Figure 1 compares the theoretical formula from Theorem 6 and its empirical estimation: we note that the results coincide and validate our formula. Moreover, 
the plot confirms that taking the $\mu$-best points leads to a lower regret than the 1-best approach.

We also compare in Fig. 2 the theoretical bounds from Theorem 9 with their empirical estimates. We remark that for $\mu \leq(1-\epsilon)^{d} \lambda$ the convergence of the two bounds to $\mathbb{E}\left(f\left(\bar{X}_{(\mu)}\right)\right)$ is fast. There exists a transition phase around $\mu \simeq(1-\epsilon)^{d} \lambda$ on which the regret is reaching a minimum: thus, one needs to choose $\mu$ both small enough to reduce bias and large enough to reduce variance. We compared to other empirically estimated values for $\mu$ from $[4,5,10]$. It turns out that if the population is large, our formula for $\mu$ leads to a smaller regret. Note that our strategy assumes that $\epsilon$ is known, which is not the case in practice. It is interesting to note that if the center of the distribution and the optimum are close (i.e. $\epsilon$ is small), one can choose a larger $\mu$ to get a lower variance on the estimator of the optimum.

\subsection{One-Shot Optimization in Nevergrad}

In this section we test different formulas and variants for the choice of $\mu$ for a larger scale of experiments in the one-shot setting. Equations 1-6 present the different formulas for $\mu$ used in our comparison.
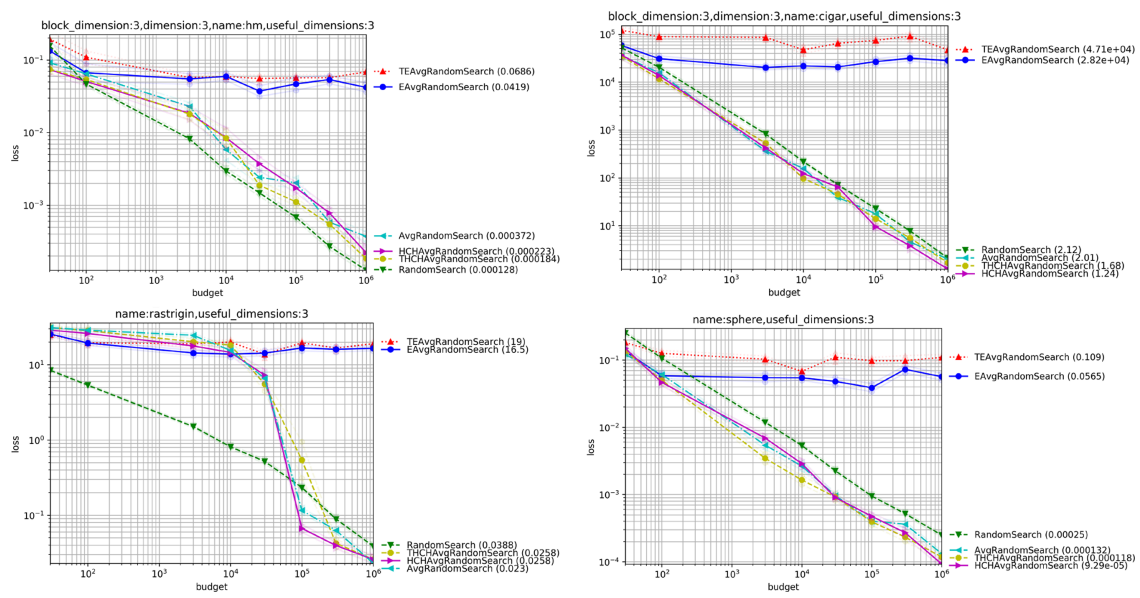

Fig. 3. Experimental curves comparing various methods for choosing $\mu$ as a function of $\lambda$ in dimension 3. Standard deviations are shown by lighter lines (close to the average lines). Each $\mathrm{x}$-axis value is computed independently. Our proposed formulas HCHAvg and THCHAvg perform well overall. See Fig. 4 for results in dimension 25 . 

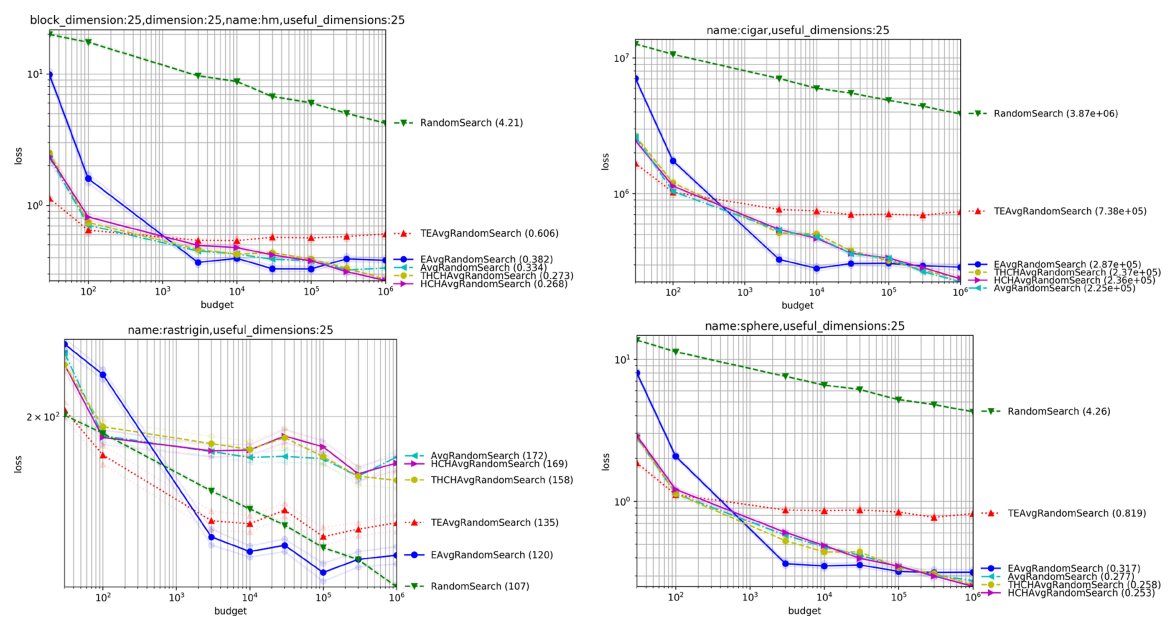

Fig. 4. Experimental curves comparing various methods for choosing $\mu$ as a function of $\lambda$ in dimension 25 (Fig. 3, continued for dimension 25; see Fig. 5 for dimension 200). Our proposals lead to good results but we notice that they are outperformed by TEAvg and EAvg for Rastrigin: it is better to not take into account non-quasi-convexity because the overall shape is more meaningful that local ruggedness. This phenomenon does not happen for the more rugged HM (Highly Multimodal) function. It also does not happen in dimension 3 or dimension 200 (previous and next figures): in those cases, THCH performed best. Confidence intervals shown in lighter color (they are quite small, and therefore they are difficult to notice).

$$
\begin{aligned}
& \mu=1 \\
& \mu=\operatorname{clip}\left(1, d, \frac{\lambda}{4}\right) \\
& \mu=\operatorname{clip}\left(1, \infty, \frac{\lambda}{1.1^{d}}\right) \\
& \mu=\operatorname{clip}\left(1, \min \left(h, \frac{\lambda}{4}\right), d+\frac{\lambda}{1.1^{d}}\right) \quad \text { Prefix: HCHAvg }(h \text { from Convex Hull }) \\
& \mu=\operatorname{clip}\left(1, \infty, \frac{\lambda}{1.01^{d}}\right) \\
& \text { Prefix: TEAvg (Tuned Exp. Avg) } \\
& \mu=\operatorname{clip}\left(1, \min \left(h, \frac{\lambda}{4}\right), d+\frac{\lambda}{1.01^{d}}\right) \quad \text { Prefix: THCHAvg (Tuned HCH Avg) }
\end{aligned}
$$

where $\operatorname{clip}(a, b, c)=\max (a, \min (b, c))$ is the projection of $c$ in $[a, b]$ and $h$ is the maximum $i$ such that, for all $j \leq i, X_{(j)}$ is on the frontier of the convex hull of $\left\{X_{(1)}, \ldots, X_{(j)}\right\}$ (Sect. 2.5). Equation 1 is the naive recommendation "pick up the best so far". Equation 2 existed before the present work: it was, until now, the best rule [16], overall, in the Nevergrad platform. Equations 3 and 5 are the proposals we deduced from Theorem 10: asymptotically on the sphere, they should have a better rate than Eq. 1. Equations 4 and 6 are counterparts of Eqs. 3 and 5 that combine the latter formulas with ideas from [16]. Theorem 
10 remains true if we add to $\mu$ some constant depending on $d$ so we fine tune our theoretical equation (Eq. 3) with the one provided by [16], so that $\mu$ is close to the value in Eq. 2 for moderate values of $\lambda$. We perform experiments in the open source platform Nevergrad [15].

While previous experiments (Figs. 1 and 2) were performed in a controlled ad hoc environment, we work here with more realistic conditions: the sampling is Gaussian (i.e. not uniform in a ball), the objective functions are not all spherelike, and budgets vary but are not asymptotic. Figures 3, 4, 5 present our results in dimension 3, 25 and 200 respectively. The objective functions are randomly translated using $\mathcal{N}\left(0,0.2 I_{d}\right)$. The objective functions are defined as $f_{\text {Sphere }}(x)=$ $\|x\|^{2}, f_{\text {Cigar }}(x)=10^{6} \sum_{i=2}^{d} x_{i}^{2}+x_{1}^{2}, f_{H M}(x)=\sum_{i=1}^{d} x_{i}^{2} \times\left(1.1+\cos \left(1 / x_{i}\right)\right)$, $f_{\text {Rastrigin }}(x)=10 d+f_{\text {sphere }}(x)-10 \sum_{i} \cos \left(2 \pi x_{i}\right)$. Our proposed equations TEAvg and EAvg are unstable: they sometimes perform excellently (e.g. everything in dimension 25, Fig. 4), but they can also fail dramatically (e.g. dimension 3, Fig. 3). Our combinations THCHAvg and HCHAvg perform well: in most settings, THCHAvg performs best. But the gap with the previously proposed Avg is not that big. The use of quasi-convexity as described in Sect. 2.5 was usually beneficial: however, in dimension 25 for the Rastrigin function, it prevented the averaging from benefiting from the overall "approximate" convexity of Rastrigin. This phenomenon did not happen for the "more" multimodal function HM, or in other dimensions for the Rastrigin function.
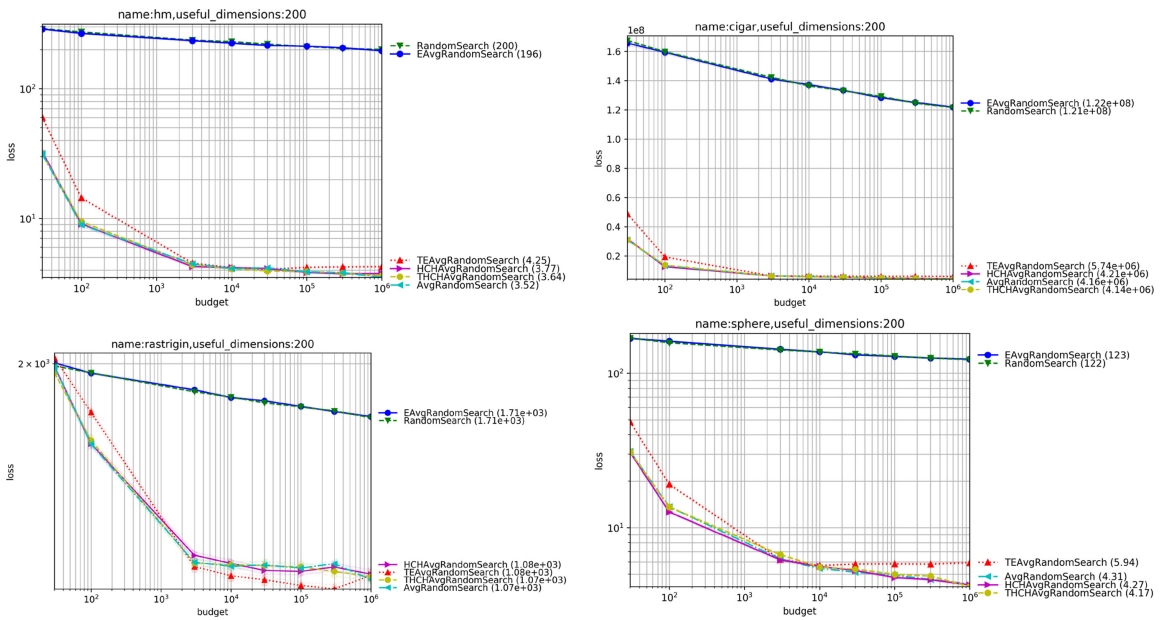

Fig. 5. Experimental curves comparing various methods for choosing $\mu$ as a function of $\lambda$ in dimension 200 (Fig. 3 and 4, continued for dimension 200). Confidence intervals shown in lighter color (they are quite small, and therefore they are difficult to notice). Our proposed methods THCHAvg and HCHAvg perform well overall. 


\section{Conclusion}

We have proved formally that the average of the $\mu$ best is better than the single best in the case of the sphere function (simple regret $O(1 / \lambda)$ instead of $O\left(1 / \lambda^{2 / d}\right)$ ) with uniform sampling. We suggested a value $\mu=\lfloor c \lambda\rfloor$ with $0<c<(1-\epsilon)^{d}$. Even better results can be obtained in practice using quasiconvexity, without losing the theoretical guarantees of the convex case on the sphere function. Our results have been successfully implemented in [15]. The improvement compared to the state of the art, albeit moderate, is obtained without any computational overhead in our method, and supported by a theoretical result.

Further Work. Our theorem is limited to a single iteration, i.e. fully parallel optimization, and to the sphere function. Experiments are positive in the convex case, encouraging more theoretical developments in this setting. We did not explore approaches based on surrogate models. Our experimental methods include an automatic choice of $\mu$ in the multimodal case using quasi-convexity, for which the theoretical analysis has yet to be fully developed - we show that this is not detrimental in the convex setting, but not that it performs better in a non-convex setting. We need an upper bound on the distance between the center of the sampling and the optimum for our results to be applicable (see parameter $\epsilon$ ): removing this need is an worthy consideration, as such a bound is rarely available in real life.

\section{References}

1. Arnold, D.V.: Optimal weighted recombination. In: Wright, A.H., Vose, M.D., De Jong, K.A., Schmitt, L.M. (eds.) FOGA 2005. LNCS, vol. 3469, pp. 215-237. Springer, Heidelberg (2005). https://doi.org/10.1007/11513575_12

2. Bergstra, J., Bengio, Y.: Random search for hyper-parameter optimization. JMLR 13, 281-305 (2012)

3. Bergstra, J., Bengio, Y.: Random search for hyper-parameter optimization. J. Mach. Learn. Res. 13, 281-305 (2012)

4. Beyer, H.G., Schwefel, H.P.: Evolution strategies -a comprehensive introduction. Natural Comput. Int. J. 1(1), 3-52 (2002). https://doi.org/10.1023/A: 1015059928466

5. Beyer, H.-G., Sendhoff, B.: Covariance matrix adaptation revisited - the CMSA evolution strategy -. In: Rudolph, G., Jansen, T., Beume, N., Lucas, S., Poloni, C. (eds.) PPSN 2008. LNCS, vol. 5199, pp. 123-132. Springer, Heidelberg (2008). https://doi.org/10.1007/978-3-540-87700-4_13

6. Bousquet, O., Gelly, S., Karol, K., Teytaud, O., Vincent, D.: Critical hyperparameters: No random, no cry (2017, preprint). https://arxiv.org/pdf/1706. 03200.pdf

7. Bubeck, S., Munos, R., Stoltz, G.: Pure exploration in multi-armed bandits problems. In: Gavaldà, R., Lugosi, G., Zeugmann, T., Zilles, S. (eds.) ALT 2009. LNCS (LNAI), vol. 5809, pp. 23-37. Springer, Heidelberg (2009). https://doi.org/10. 1007/978-3-642-04414-4_7

8. Escalante, H., Reyes, A.M.: Evolution strategies. CCC-INAOE tutorial (2013) 
9. Fournier, H., Teytaud, O.: Lower bounds for comparison based evolution strategies using VC-dimension and sign patterns. Algorithmica (2010)

10. Hansen, N., Ostermeier, A.: Completely derandomized self-adaptation in evolution strategies. Evol. Comput. 11(1), 159-195 (2003)

11. Hansen, N., Arnold, D.V., Auger, A.: Evolution strategies. In: Kacprzyk, J., Pedrycz, W. (eds.) Springer Handbook of Computational Intelligence, pp. 871898. Springer, Heidelberg (2015). https://doi.org/10.1007/978-3-662-43505-2_44

12. Jebalia, M., Auger, A.: Log-linear convergence of the scale-invariant $\left(\mu / \mu_{w \lambda}\right)$-ES and optimal $\mu$ for intermediate recombination for large population sizes. In: Schaefer, R., Cotta, C., Kołodziej, J., Rudolph, G. (eds.) PPSN 2010. LNCS, vol. 6238, pp. 52-62. Springer, Heidelberg (2010). https://doi.org/10.1007/978-3-642-15844$5 \_6$

13. McKay, M.D., Beckman, R.J., Conover, W.J.: A comparison of three methods for selecting values of input variables in the analysis of output from a computer code. Technometrics 21(2), 239-245 (1979)

14. Niederreiter, H.: Random Number Generation and quasi-Monte Carlo Methods. Society for Industrial and Applied Mathematics, Philadelphia (1992)

15. Rapin, J., Teytaud, O.: Nevergrad - A gradient-free optimization platform. https:// GitHub.com/FacebookResearch/Nevergrad (2018)

16. Teytaud, F.: A new selection ratio for large population sizes. In: Di Chio, C., et al. (eds.) EvoApplications 2010. LNCS, vol. 6024, pp. 452-460. Springer, Heidelberg (2010). https://doi.org/10.1007/978-3-642-12239-2_47

17. Teytaud, F., Teytaud, O.: Why one must use reweighting in estimation of distribution algorithms. In: Genetic and Evolutionary Computation Conference, GECCO 2009, Proceedings, Montreal, Québec, Canada, 8-12 July 2009, pp. 453-460 (2009) 